ARTÍCULO DE INVESTIGACIÓN

\title{
Hipótesis de Naturalización de Darwin evaluada en la flora exótica de Chile continental
}

\author{
Darwin's Naturalization Hypothesis assessed in the alien flora of continental Chile
}

\author{
VÍCTOR M. ESCOBEDO ${ }^{1}$, JORGE E. ARANDA ${ }^{1,2}$ \& SERGIO A. CASTRO $1,2,3$, * \\ ${ }^{1}$ Laboratorio de Ecología y Biodiversidad, Universidad de Santiago de Chile, Alameda Bernardo O’Higgins 3363, Santiago, Chile \\ ${ }^{2}$ Centro para el Desarrollo de la Nanociencia y Nanotecnología (CEDENNA) 917-0124, Santiago, Chile \\ ${ }^{3}$ Centro de Estudios Avanzados en Ecología y Biodiversidad (CASEB), Pontificia Universidad Católica de Chile, Santiago, Chile \\ ${ }^{*}$ Correspondencia: sergio.castro@usach.cl
}

\begin{abstract}
RESUMEN
La Hipótesis de Naturalización de Darwin (HND) plantea que el éxito de naturalización de especies exóticas se ve favorecido cuando el parentesco filogenético entre el colonizador y la comunidad recipiente es distante, debido a que esta situación reduciría la intensidad de la competencia entre especies. Inversamente, especies cercanas filogenéticamente mantendrían alta intensidad de competencia, reduciendo la probabilidad de naturalización. Desde el punto de vista poblacional, el concepto de naturalización es dinámico e involucra dos componentes: el aumento progresivo del tamaño y la distribución espacial de la población invasiva. En el presente estudio, centramos nuestra atención en el componente espacial de la naturalización, evaluando el papel del parentesco filogenético como determinante de la naturalización en la flora exótica presente en Chile continental. Siguiendo la predicción de la HND, sería posible esperar que aquellas especies exóticas emparentadas con la flora nativa debiesen mostrar rangos distribucionales menos extensos que taxa exóticas distantes filogenéticamente. Utilizando el ordenamiento taxonómico APG III como indicador del parentesco filogenético, nuestro análisis mostró que taxa exóticas congenéricas a taxa nativas tienen rangos distribucionales en promedio más extensos que aquellos de parentesco más distante (i.e. especies que pertenecen a familias u órdenes no representados en la flora nativa del país). Estos hallazgos no apoyan la HND ya que las taxa más distantes muestran rangos más reducidos. Los resultados sugieren que el parentesco filogenético podría haber facilitado la naturalización de plantas exóticas en Chile continental, al menos para especies que pertenecen a géneros y familias ya representados en la flora nativa.
\end{abstract}

Palabras clave: flora exótica, flora nativa, invasiones biológicas, parentesco filogenético, rango geográfico.

\begin{abstract}
The Darwin's Naturalization Hypothesis (DNH) states that the successful naturalization of alien species is favored when the phylogenetic relationship between the colonizer and the recipient community is distant. Conversely, related species would compete strongly, hence reducing the probability of naturalization. From a populational perspective, the concept of naturalization involves both the progressive increase in size and spatial distribution of the invasive population. In this study, we focused our attention on the spatial component of naturalization, assessing the role of phylogenetic relatedness as a determinant of its extension. Following the DNH, it is expected that those alien species closely related to the native flora would show narrower distribution ranges than alien taxa less related to native species. Using the APG III taxonomic system as an indicator of phylogenetic relationships in the vascular flora of continental Chile, our analysis showed that alien species with congeneric native counterparts have larger ranges than alien species with a distant relationship (i.e. species belonging to families or orders not represented in the native flora). These results do not support the DNH because the more distant taxa (less related species) show distributional ranges smaller. Results suggest that close relatedness could have facilitated the naturalization of alien plants in continental Chile, at least for species belonging to genus and families represented on the native flora.
\end{abstract}

Key words: alien flora, biological invasion, geographic range, native flora, phylogenetic relatedness.

\section{INTRODUCCIÓN}

Las invasiones biológicas han cautivado la atención de ecólogos y biogeógrafos modernos
(Pyšek et al. 2006) debido a que protagonizan un papel importante como componente del cambio global y de la ecología de los ecosistemas expuestos a la globalización 
(D’Antonio \& Vitousek 1992, Vitousek 1994, Vitousek et al. 1997, Callaway \& Maron 2006). Actualmente, organismos pertenecientes a diversos grupos taxonómicos son translocados desde una región a otra, con la que no comparten historia previa (Williamson 1996, Davis 2009). Aunque se estima que la mayor parte de los organismos que inician esta dispersión no llegan a establecerse exitosamente en el área de destino, en ocasiones un reducido número de propágulos pueden configurar una colonia fundadora y naturalizarse (Kolar \& Lodge 2001, Sakai et al. 2001). Uno de los desafíos centrales en el estudio de las invasiones biológicas ha sido comprender qué factores determinan este proceso de naturalización (Williamson 1996, Lockwood et al. 2007, Davis 2009), entendido como un evento de expansión poblacional (ver más abajo).

Diversas hipótesis se han propuesto para explicar por qué algunas especies son capaces de naturalizarse y otras no (Kolar \& Lodge 2001, Mitchell et al. 2006, Castro et al. 2007). Un papel particularmente intrigante lo tiene la llamada Hipótesis de Naturalización de Darwin (HND; Daehler 2001). Esta hipótesis planteada originalmente por Charles Darwin (1859), supone que el éxito de naturalización está influenciado por el parentesco filogenético entre el colonizador y los miembros de la comunidad recipiente (Chesson 2000, Adler et al. 2007, Procheş et al. 2008, Macdougall et al. 2009, Jiang et al. 2010, Thuiller et al. 2010). En este contexto, especies invasoras que exhiban un estrecho parentesco filogenético con la comunidad recipiente deberían exhibir una alta sobreposición de nicho, generando de esta manera una alta intensidad de competencia con los miembros de la comunidad recipiente (Cahill et al. 2008, Cavender-Bares et al. 2009, Mayfield \& Levine 2010). Por tanto, bajo condiciones de mayor parentesco filogenético con la comunidad recipiente, un colonizador tendría menor probabilidad de naturalizarse. Inversamente, cuando el colonizador tiene un bajo nivel de parentesco con los miembros de la comunidad, la HND plantea que la intensidad competitiva disminuiría, hecho que contribuirá a facilitar la naturalización (Darwin 1859, Daehler 2001, Adler et al. 2007, Macdougall et al. 2009, Jiang et al. 2010, Mayfield \& Levine 2010).
Aunque existen diversas concepciones de "naturalización" (Richardson et al. 2000), desde el punto de vista poblacional puede ser visualizado como un proceso en que una colonia de una especie exótica introducida, disponiendo de mecanismos reproductivos independientes de la asistencia humana, conforma una población que crece en tamaño y se expande (Sakai et al. 2000, Shigesada \& Kawasaki 2001). Así, dependiendo de los niveles de abundancia y distribución, es posible reconocer diversos estados de avance en el proceso de naturalización (Shigesada \& Kawasaki 2001). Por tanto, si el parentesco filogenético determina el componente distribucional de naturalización -como lo sugiere la HDN- sería posible predecir que aquellas especies exóticas emparentadas con los miembros de la comunidad recipiente debiesen mostrar rangos distribucionales de menor tamaño en comparación a aquellos taxa menos emparentados. Interesantemente, aunque un reducido número de estudios han evaluado formalmente la Hipótesis de Naturalización de Darwin, hasta la fecha ninguno de ellos se ha focalizado sobre el componente distribucional (i.e. tamaño del rango) implícito en la naturalización (ver Procheş et al. 2008). Probablemente, esta desatención se pueda explicar por no disponer de información que permita controlar el efecto de otros factores (i.e. tiempo mínimo de residencia, origen biogeográfico y escala filogenética y espacial, entre otros) que determinan el rango y que pueden oscurecer la señal filogenética (ver Procheş et al. 2008).

Dado que el rango distribucional constituye uno de los componentes del proceso de naturalización, el objetivo del presente estudio es evaluar la Hipótesis de Naturalización de Darwin y su efecto sobre el rango distribucional de plantas exóticas en Chile continental. Para ello, caracterizamos el tamaño del rango en plantas vasculares exóticas que difieren en el nivel de parentesco filogenético respecto de la flora nativa, controlando el presunto efecto de factores covariantes tales como el tiempo mínimo de residencia (TMR; sensu Wu et al. 2003) y el origen biogeográfico (Di Castri 1989, Thebaud \& Simberloff 2001). Estos factores han sido sugeridos como determinantes de la naturalización y extensión del rango para la flora exótica de Chile (ver Arroyo et al. 2000, Castro et al. 2005). En consecuencia, para un 
gradiente de parentesco filogenético y si la HDN es correcta, se esperaría que especies exóticas distantes filogenéticamente de la flora nativa de Chile muestren tamaños de rango mayores que aquellas especies más emparentadas.

\section{METODOS}

\section{Flora naturalizada en Chile continental}

La flora exótica naturalizada en Chile se encuentra representada por unas 748 especies de angiospermas (http://i3n.usach.cl/filt_especies.asp), distribuidas principalmente en la zona central del país (Marticorena \& Quezada 1985, Matthei 1995, Figueroa et al. 2004). Para un subconjunto de 419 especies, se logró conformar una base de datos conteniendo información de su distribución geográfica, tiempo mínimo de residencia y origen biogeográfico. Adicionalmente, para cada una de estas especies se estableció su estatus taxonómico hasta el nivel de Orden, de acuerdo a las indicaciones nomenclaturales del APG III (APG III 2009; www.mobot. org/MOBOT/research/APweb).

En términos geopolíticos, Chile se dispone como una estrecha franja de territorio relativamente aislado del resto de Sudamérica por barreras naturales (Jaksic 1998, Arroyo et al. 1999), de extenso recorrido latitudinal y cuyas regiones administrativas se disponen secuencialmente en sentido norte-sur (Fig. 1A). Colectas de herbario y publicaciones han documentado la presencia/ausencia regional de flora exótica, información que permitió establecer la distribución geográfica y rango para las 419 taxa estudiadas. A partir de esta información, se estableció el número total de regiones administrativas ocupadas por cada una de las especies, y luego se determinó la extensión latitudinal (en kilómetros) de este rango. Para estos efectos, el rango distribucional se estimó como la sumatoria de la longitud máxima de cada región administrativa ocupada (IGM 2010); este procedimiento supone que cada especie se distribuye a lo largo de cada región (ver Castro et al. 2005).

Diversos autores han indicado que algunos factores pueden afectar el rango distribucional de especies exóticas (Daehler 1988, Di Castri 1989, Groves 1991, Theoharides \& Dukes 2007, Hulme 2009). Entre las variables o factores documentados que alteran el rango de plantas exóticas se encuentran el tiempo mínimo de residencia y el origen biogeográfico (Rozenfields \& Mackenzie 1999, Pyšek et al. 2003). El tiempo mínimo de residencia informa el lapso de tiempo transcurrido desde que una especie es documentada por primera vez en el territorio hasta el presente (sensu Castro et al. 2005). En términos operacionales, el tiempo mínimo de residencia fue establecido a partir del primer registro botánico realizado en el territorio, información obtenida a partir del examen de publicaciones históricas y colectas (véase Castro et al. 2005). A cada especie se le asignó una de las categorías de tiempo mínimo de
(A)

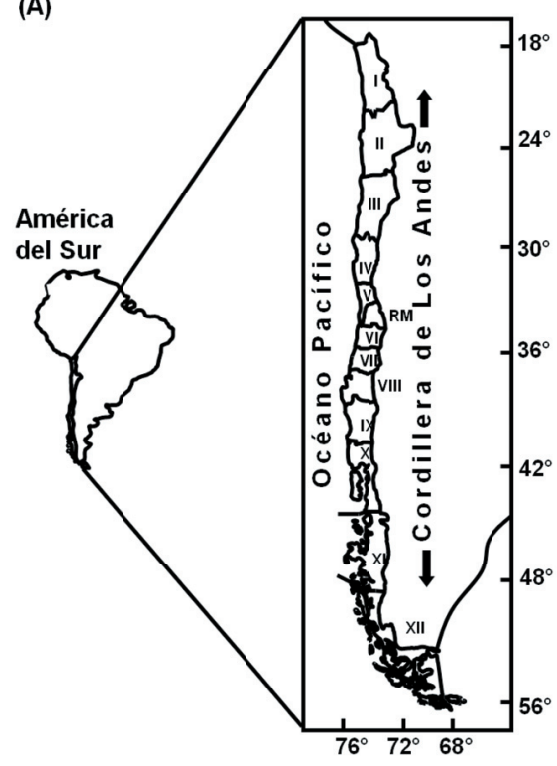

(B)

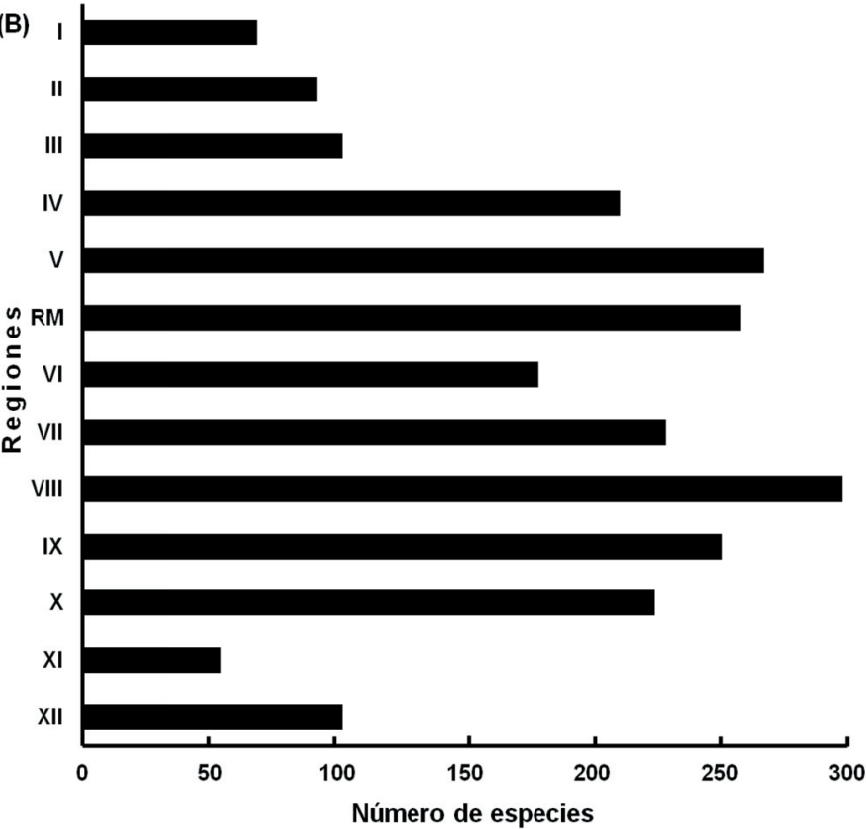

Fig. 1: (A) Localización geográfica y regionalización política de Chile continental. Las regiones están identificadas con números desde I hasta XII, y con la sigla RM para la Región Metropolitana. (B) Distribución regional de las 419 especies de plantas exóticas estudiadas, presentes en cada región administrativa de Chile continental.

(A) Geographical location and political regionalization of continental Chile. The regions are identified by roman numbers (from I to XII; RM for Metropolitan Region). (B) Regional distribution of the 419 alien plants studied, present in each administrative region of continental Chile. 
residencia (véase sección de Análisis), las que fueron determinadas por períodos de 50 años a partir del año 1800. Especies introducidas con anterioridad a este año fueron agrupadas en una sola categoría.

Debido a que la mayor parte de la flora introducida en Chile es de origen europeo, particularmente de la región mediterránea (Montenegro et al. 1991), algunos autores han planteado que la homología climática habría facilitado su naturalización (Di Castri 1989, Matthei 1995, Arroyo et al. 2000). Por esta razón, a través de una revisión bibliográfica compilamos información acerca del origen de cada una de las especies estudiadas, lo que fue registrado como un segundo cofactor asociado al tamaño del rango. Para cada taxa, la información fue codificada de acuerdo a su procedencia continental, como planta europea, sudamericana, africana, asiática y de Oceanía.

Finalmente, para establecer el nivel de parentesco filogenético de las taxa exóticas respecto de la comunidad residente utilizamos un gradiente taxonómico de tres niveles. En el primer nivel (grupo Cercano) se consideraron aquellas especies pertenecientes a un género representado en la flora nativa de Chile, agrupación considerada de alta proximidad filogenética. Un segundo nivel (grupo Intermedio) fue establecido por el conjunto de especies que pertenecen a géneros no representados en la flora nativa pero que pertenecen a familias que sí lo están. Por último, en un tercer y más distante nivel (grupo Distante) de parentesco se agruparon aquellas taxa que pertenecen a géneros y familias no representadas en la flora nativa de Chile.

\section{Análisis}

Para evaluar la HND realizamos un análisis de varianza (ANOVA) de tres vías, donde los factores fueron parentesco filogenético, tiempo mínimo de residencia y origen biogeográfico; y como variable dependiente se utilizó el tamaño de rango (en km), previa normalización mediante logaritmo natural. En el factor de parentesco filogenético se reconocieron tres niveles: Cercano, Intermedio y Distante; mientras que en el factor de tiempo mínimo de residencia se reconocieron cinco niveles: (a) $\mathrm{TMR} \geq 210$ años (i.e. primer registro botánico previo al año 1800); (b) $209 \geq$ TMR $\geq 160$ años (i.e. primer registro botánico entre los años 1801 y 1850); (c) $159 \geq \mathrm{TMR} \geq 110$ años (i.e. primer registro botánico entre los años 1851 y 1900); (d) $109 \geq$ TMR $\geq 60$ (i.e. primer registro entre los años 1901 y 1950) y (e) $59 \geq$ TMR $\geq 10$ años (i.e. primer registro entre los años 1951 - 2000). Finalmente, para el factor de origen biogeográfico se reconocieron seis niveles: (a) Europa; (b) América; (c) África; (d) Asia; (e) Oceanía y (f) dos o más continentes, considero como origen mixto.

El ANOVA fue realizado utilizando el programa $R$ (R Development Core Team 2010), con aplicación de la prueba a posteriori de Tukey. Debido al desbalance de nuestros datos no fue posible analizar las interacciones entre los factores considerados.

\section{RESULTADOS}

\section{Distribución y rango de la flora exótica}

De las 419 especies analizadas, la mayor parte de ellas se distribuyeron en la porción central de Chile continental (Fig. 1B). De hecho, entre la IV y VIII Región de Chile (Chile central) se acumuló el $91.2 \%$ de las 419 especies, representación que disminuyó al 36.3 \% y $72.5 \%$ para las regiones extremas del norte (entre la I y la III Región) y sur (entre la IX y la XII Región), respectivamente. Por otra parte, el análisis total de las especies estudiadas mostró que la distribución de tamaños de rango osciló entre un mínimo de $137 \mathrm{~km}$ y un máximo de $4135 \mathrm{~km}$. En términos generales, la distribución de tamaños de rango mostró una alta concentración de especies $(n=252)$ variando entre 130 y $2000 \mathrm{~km}$ (Fig. 2), y una menor cantidad de especies ( $\mathrm{n}=$ 167) entre 2000 y 4500 km (Fig. 2).

De las 419 especies registradas en nuestro estudio, solo 24 tuvieron un tiempo mínimo de residencia $>210$ años, exhibiendo un rango promedio de $2299 \mathrm{~km}$ (Tabla 1). Aquellas taxa que tuvieron un TMR entre 209 y 160 años

\section{TABLA 1}

Parámetros del tamaño de rango geográfico $(\mathrm{X} \pm$ DE; km) para 419 especies de plantas exóticas, presentes en Chile continental. Esta información ha sido organizada de acuerdo al tiempo mínimo de residencia y origen biogeográfico.

Parameters $(\mathrm{X} \pm \mathrm{SD} ; \mathrm{km})$ of size of geographic range for 419 species of alien plants inhabiting continental Chile. This information is organized according to the minimum residence time and biogeographic origin.

\begin{tabular}{|c|c|c|}
\hline Factores & $\mathrm{X} \pm \mathrm{DE}(\mathrm{km})$ & $\mathrm{N}$ \\
\hline \multicolumn{3}{|c|}{ Tiempo mínimo de residencia } \\
\hline$>210$ años & $2.299 \pm 805$ & 24 \\
\hline $209-160$ años & $2.202 \pm 1.128$ & 94 \\
\hline $159-110$ años & $2.113 \pm 1.096$ & 119 \\
\hline $109-60$ años & $1.588 \pm 1.017$ & 87 \\
\hline $59-10$ años & $886 \pm 725$ & 95 \\
\hline \multicolumn{3}{|c|}{ Origen biogeográfico } \\
\hline Europa & $1.799 \pm 1.133$ & 312 \\
\hline América & $1.635 \pm 1.063$ & 52 \\
\hline África & $1.366 \pm 1.067$ & 13 \\
\hline Asia & $1.666 \pm 1.354$ & 10 \\
\hline Oceanía & $1.347 \pm 415$ & 3 \\
\hline Mixto & $1.768 \pm 1.126$ & 29 \\
\hline
\end{tabular}


estuvieron representados por 94 especies y mostraron un tamaño de rango distribucional promedio de $2202 \mathrm{~km}$ (Tabla 1). Así mismo, 119 especies tuvieron un TMR entre 159 y 110 años y un rango distribucional promedio de 2113 $\mathrm{km}$ (Tabla 1). Mientras que otras 87 especies tuvieron un TMR entre 109 y 60 años, con un tamaño de rango distribucional promedio de 1588 km (Tabla 1). Finalmente, 95 especies tuvieron un TMR entre 59 y 10 años, con un rango distribucional promedio de $886 \mathrm{~km}$ (Tabla 1).

Por otra parte, 312 especies tuvieron origen europeo, cuyo rango distribucional promedió los $1799 \mathrm{~km}$ (Tabla 1). Otras 52 taxa tuvieron origen americano, con un tamaño de rango promedio de $1635 \mathrm{~km}$ (Tabla 1). Las especies africanas fueron 13, y tuvieron en promedio un rango distribucional de $1366 \mathrm{~km}$ (Tabla 1). Diez plantas tuvieron origen asiático y promediaron un rango distribucional de $1666 \mathrm{~km}$, mientras que otras tres especies que proceden de Oceanía mostraron un rango distribucional promedio de $1347 \mathrm{~km}$ (Tabla 1). Finalmente, las taxa de origen mixto (presentes en dos o más continentes) fueron 29 especies, con un rango distribucional promedio de $1768 \mathrm{~km}$ (Tabla 1).

\section{HND y rango de distribución}

E1 40.3 \% de las especies exóticas analizadas perteneció a géneros ya representados en la flora nativa de Chile continental. El 56.3 \% perteneció a familias ya representadas en la flora nativa; mientras que aquellas especies no emparentadas a nivel de género o familia representaron solo el $3.4 \%$ de la flora exótica. El conjunto de especies con mayor nivel de parentesco filogenético (Cercano) a la flora nativa (i.e. especies congenéricas) tuvo un rango distribucional promedio de 1936 $\mathrm{km}$, mientras que el nivel Intermedio (i.e. especies confamiliares) mostró un rango de distribución promedio de $1682 \mathrm{~km}$ (Fig. $3)$. Aquellas especies distantes, con menor nivel de parentesco (i.e. pertenecientes a géneros y familias no representadas en la flora nativa) mostraron tamaños de rango en promedio de $842 \mathrm{~km}$ (ver Fig. 3). De esta manera, el parentesco filogenético mostró un efecto significativo sobre el tamaño de rango distribucional para los niveles de parentesco Cercano e Intermedio, aun controlando el efecto de los cofactores (Tabla 2). Estas diferencias estuvieron determinadas por rangos significativamente más extensos en aquellas especies pertenecientes a géneros y familias ya representados en la flora nativa (Prueba de Tukey, $\mathrm{D}=3.3 ; \mathrm{P}=0.003$; ver Fig. 3). El ANOVA también mostró un efecto positivo y significativo del TMR sobre el tamaño del rango distribucional (Tabla 2), ya que especies introducidas hace más tiempo exhibieron una mayor extensión del rango que aquellas introducidas recientemente (Tabla 1). A diferencia del caso anterior, el origen

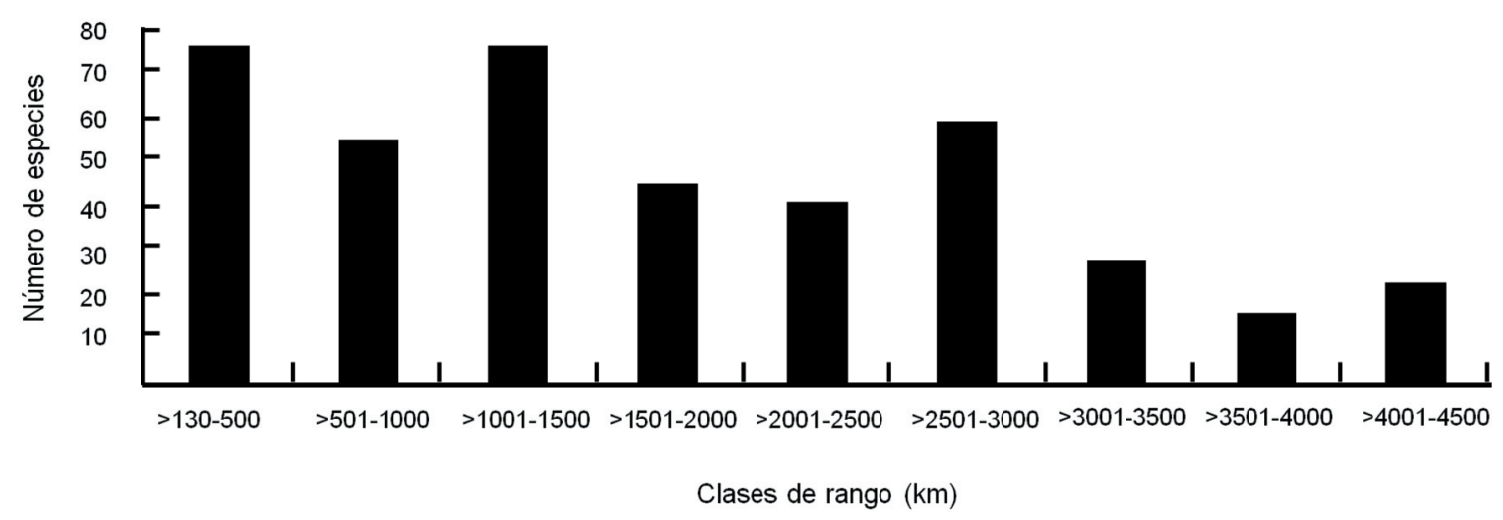

Fig. 2: Distribución de los tamaños de rango geográfico $(\mathrm{km})$ para las 419 especies de plantas exóticas estudiadas, presentes en Chile continental. Las clases han sido definidas cada $500 \mathrm{~km}$, a excepción del primer rango de clases cuyo mínimo valor fue $137 \mathrm{~km}$.

Distribution of geographic range sizes $(\mathrm{km})$ for 419 alien plant species, present in continental Chile. The classes have been defined each $500 \mathrm{~km}$, except for the first class whose minimum value was $137 \mathrm{~km}$. 
biogeográfico no mostró efectos significativos sobre el tamaño del rango (Tabla 2).

\section{DISCUSIÓN}

Nuestros resultados mostraron que el tamaño del rango distribucional es mayor para aquellas especies emparentadas con taxa nativos, a nivel de género (parentesco Cercano) y familia (parentesco Intermedio). Interesantemente, esta relación fue obtenida aun controlando el efecto de factores covariantes como el tiempo mínimo de residencia y el origen biogeográfico, que han sido señalados en la literatura como determinantes del tamaño del rango en plantas exóticas (Arroyo et al. 2000, Rejmánek 2000, Pyšek et al. 2003, Castro et al. 2005). En consecuencia, nuestros resultados no apoyan la Hipótesis de Naturalización de Darwin como explicación del componente distribucional de la naturalización en plantas exóticas de Chile continental.

Pese a que la HND fue formulada hace más de 150 años, a la fecha existe un reducido número de estudios que la han puesto a prueba. De la evidencia disponible, algunos han resultado en apoyo (Rejmánek 1996, Ricciardi \& Atkinson 2004, Strauss et al. 2006, Jiang et al. 2010, Schaefer et al. 2011) mientras que otros estudios han desestimado su importancia (Daehler 2001, Duncan \& William 2002, Lambdon \& Hulme 2006, Ricciardi \& Mottiar 2006). Esta discrepancia ha encendido la controversia acerca del papel del parentesco filogenético en determinar el éxito de la naturalización (Diez et al. 2008, 2009, Thuiller et al. 2010). Aunque originalmente la HND plantea que la naturalización se vería

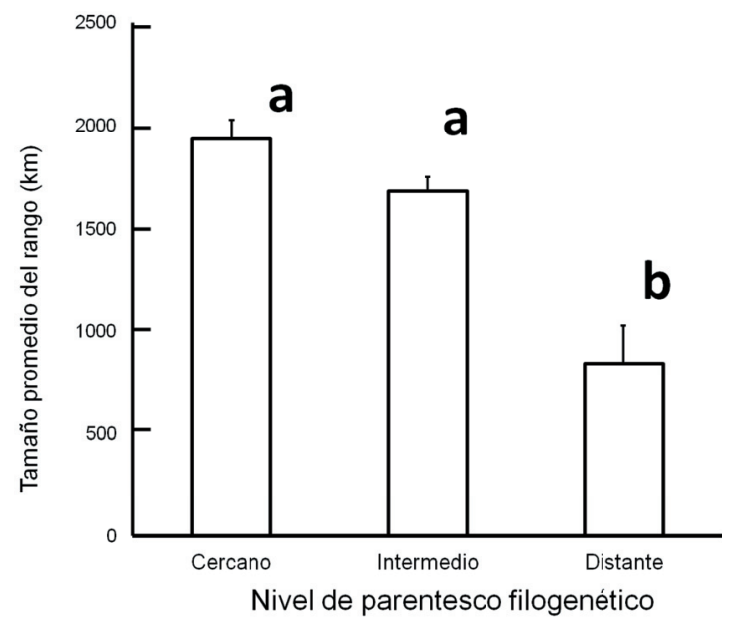

Fig. 3: Tamaño de rango promedio $(\mathrm{X} \pm \mathrm{EE})$ para las 419 especies de plantas exóticas estudiadas en Chile continental. De acuerdo a su parentesco filogenético, las plantas han sido agrupadas en tres categorías: Cercano ( $\mathrm{n}=169$ especies), Intermedio ( $\mathrm{n}=236$ especies) y Distante ( $\mathrm{n}=14$ especies) (ver detalles en Metodología). Las letras A y B identifican diferencias significativas entre los niveles de parentesco filogenético, de acuerdo a la prueba de Tukey.

Mean $(\mathrm{X} \pm \mathrm{SE})$ range size for 419 alien plant species present in continental Chile. According to their phylogenetic relationship, plants have been grouped into three categories: Close $(\mathrm{n}=169$ species), Intermediate $(\mathrm{n}=236$ species $)$ and Distant ( $\mathrm{n}=14$ species) (see details in Methods). The letters $\mathrm{A}$ and B identify significant differences among phylogenetic levels, according to the Tukey test.

TABLA 2

ANOVA de tres vías para el tiempo mínimo de residencia, origen biogeográfico y parentesco filogenético, como determinantes del tamaño del rango distribucional de las especies de plantas exóticas de Chile continental.

Three-way ANOVA for the minimum residence time, biogeographic origin and phylogenetic relatedness as determinants of range size for alien flora inhabiting continental Chile.

\begin{tabular}{lccccc}
\hline Factores & GL & SC & CM & F & P \\
\hline Parentesco filogenético & 2 & 12.7 & 6.3 & 11.4 & $<0.0001$ \\
Tiempo mínimo de residencia & 4 & 69.7 & 17.4 & 31.3 & $<0.0001$ \\
Origen biogeográfico & 5 & 0.9 & 0.1 & 0.3 & 0.893 \\
Residuos & 407 & 226.6 & 0.5 & & \\
Total & 418 & 310.1 & & & \\
\hline
\end{tabular}


favorecida cuando el parentesco entre especies colonizadoras y la comunidad recipiente es distante, fue el mismo Darwin quien también indicó que la cercanía filogenética podría facilitar la naturalización (Darwin 1859). Esto se explicaría porque especies exóticas filogenéticamente cercanas a miembros de la comunidad recipiente mostrarían atributos biológicos similares a sus parientes, y en consecuencia, funcionalmente preadaptados a las condiciones ambientales de dicha comunidad (Diez et al. 2008). Esta aparente discrepancia dio origen al Dilema de la Naturalización de Darwin (sensu Diez et al. 2008). En este escenario, nuestros resultados parecen obedecer -al menos parcialmente- a este último planteamiento, puesto que aquellos taxa exóticos emparentados con miembros de la flora nativa de Chile continental (a nivel de género y familia), mostraron en promedio mayor extensión de rango distribucional que aquellos taxa menos emparentados.

En efecto, de acuerdo a nuestros hallazgos es posible especular que la cercanía filogenética entre las especies exóticas y nativas conferiría a las primeras la posesión de rasgos morfofuncionales homólogos y en cierto modo "preadaptados", hecho que facilitaría la expansión geográfica de taxa exóticos estrechamente emparentados (Bruno et al. 2003, Wiens \& Graham 2005). Dos líneas de evidencia indirecta pueden ser esgrimidas para apoyar esta última hipótesis. Por una parte, Diez et al. (2008), Jiang et al. (2010) y Thuiller et al. (2010) han indicado que los mecanismos asociados a la HND (i.e. competencia interespecífica) tienen mayor importancia en escalas espaciales reducidas (i.e. $\mathrm{m}^{2}$ ), y no a escala geográfica (i.e. $\mathrm{km}^{2}$ ). Estos autores señalaron que a nivel geográfico, las condiciones macroambientales (clima, precipitaciones, temperatura, etc.) tienen mayor relevancia al momento de determinar la extensión del rango, incluso por sobre la facilitación antrópica a la expansión espacial e interacciones interespecíficas (ver también Chesson 2000, Hubbell 2001, McKinney 2006, Sax et al. 2007). Si bien un estrecho parentesco filogenético puede estar relacionado con la posesión de atributos preadaptados, esto no excluye la posibilidad de que taxa más distantes filogenéticamente no puedan naturalizarse (Procheş et al. 2008).
Adicionalmente, Castro \& Jaksic (2008a, 2008b) han documentado que el patrón de recambio geográfico exhibido por plantas exóticas es similar al mostrado por las especies nativas en Chile continental. Aunque estos autores no analizaron el componente filogenético, sus resultados también sugieren que las condiciones ambientales a lo largo del gradiente latitudinal podrían determinar la expansión de taxa exóticos del mismo modo como ocurre con los taxa nativos (ver también Sax 2001). No obstante, el papel del parentesco filogenético como determinante de la expansión en especies exóticas requiere un examen más detallado para el caso de la flora exótica de Chile continental. En este sentido, resulta clave la comparación directa entre los tamaños de rango de especies nativas y exóticas emparentadas. Si la hipótesis preadaptativa es correcta, se debería observar una correlación positiva entre el tamaño de rango de especies exóticas y nativas pertenecientes al mismo género o familia.

El proceso de naturalización conlleva dos componentes poblacionales acoplados, el crecimiento poblacional y la expansión del rango (Richardson et al. 2000, Shigesada \& Kawasaki 2001). Pese a que ambas variables pueden ser utilizadas como indicadoras de naturalización, hasta la fecha los estudios que han evaluado la HND solo se han centrado en aspectos relativos a la abundancia (Thuiller et al. 2010). Por ello, una perspectiva novedosa de nuestro trabajo es el uso del rango geográfico como expresión del proceso de naturalización (Richardson et al. 2000). La inclusión de factores covariantes que puedan modificar la capacidad de detección de la señal filogenética es un segundo aspecto novedoso de nuestro estudio. Estos antecedentes sugieren que la inclusión de covariables asociadas a la abundancia poblacional de especies exóticas representa un procedimiento necesario de implementar, pues podría constituir una importante fuente de las discrepancias emergidas en los estudios que hasta la fecha han evaluado la HND.

Las especies naturalizadas pueden infligir importantes impactos ecológicos, ambientales y sociales (Vilá et al. 2009). Por esta razón, es de interés desarrollar estrategias tendientes a predecir qué especies pueden naturalizarse y transformarse en malezas (Mack 1996, Mack 
et al. 2000, Maris \& Béchet 2010). En Chile continental la detección temprana de plantas exóticas constituye una de las estrategias que es necesario fortalecer (CONAMA 2003). Así, nuestros resultados pueden apoyar futuras medidas de control temprano de especies de plantas introducidas al territorio. En esta línea, nuestros hallazgos sugieren que la cercanía filogenética entre plantas exóticas -al menos al nivel de género y familia- podría ser un buen predictor de la expansión de rango distribucional en especies que aún no han sido introducidas en Chile.

En resumen, el tamaño del rango distribucional de la flora exótica en Chile continental es mayor para aquellas especies emparentadas con taxa nativos, a nivel de género o familia, y menor para aquellas especies que no pertenecen a estas categorías taxonómicas. Este hecho podría ser explicado por la presencia de rasgos homólogos, preadaptados que facilitan la expansión de la flora emparentada, hecho que no apoya la Hipótesis de Naturalización de Darwin.

AGRADECIMIENTOS: Financiaron parcialmente esta investigación el proyecto Fondecyt $\mathrm{N}^{\circ}$ 11085013; la Línea 8 del Centro para el Desarrollo de la Nanociencia y la Nanotecnología (CEDENNA) de la Universidad de Santiago de Chile; el Centro para el Estudio Avanzado en Ecología y Biodiversidad (CASEB) de la Pontificia Universidad Católica de Chile. Agradecemos al curso de invasiones biológicas impartido por Fabián Jaksic (primer semestre de 2011; Departamento de Ecología, Pontificia Universidad Católica de Chile), desde donde obtuvimos inspiración para realizar este trabajo. Agradecemos la revisión crítica y los aportes de Ernesto Gianoli y dos revisores anónimos, cuya revisión y comentarios contribuyeron a mejorar nuestro manuscrito.

\section{LITERATURA CITADA}

ADLER PB, J HILLE RIS LAMBERS \& JM LEVINE (2007) A niche for neutrality. Ecology Letters 10: 95-104.

APG III (2009) An update of the angiosperm phylogeny group classification for the orders and families of flowering plants: APG III. Botanical Journal of the Linnean Society 161: 105-121.

ARROYO MTK, R ROZZI, JA SIMONETTI, P MARQUET \& M SALLABERRY (1999) Central Chile. En: Mittermeier RA, N Myers, P Robles-Gil \& C Goettsch-Mittermeier (eds) Hotspots: Earth's biologically richest and most endangered terrestrial ecosystems: 161-171. Cemex, Conservation International, Ciudad de Mexico.

ARROYO MTK, C MARTICORENA, O MATTHEI \& L CAVIERES (2000) Plant invasions in Chile: Present patterns and future predictions. En: Mooney HA \& RJ Hobbs (eds) Invasive species in a changing world: 385-421. Island Press, Covelo, California.

BRUNO JF, JJ STACHOWICZ \& MD BERTNESS (2003) Inclusion of facilitation into ecological theory. Trends in Ecology and Evolution 18: 119-125.

CAHILL JF, SW KEMBEL, EG LAMB \& P KEDDY (2008) Does phylogenetic relatedness influence the strength of competition among vascular plants? Perspectives in Plant Ecology, Evolution and Systematics 10: 41-50.

CALLAWAY R \& JL MARON (2006) What have exotic plant invasions taught us over the past 20 years? Trends in Ecology and Evolution 21: 369-374.

CASTRO SA, JA FIGUEROA, M MUÑOZ-SCHICK \& FM JAKSIC (2005) Minimum residence time, biogeographical origin, and life cycle as determinants of the geographical extent of naturalized plants in continental Chile. Diversity and Distributions 11: 183-191.

CASTRO SA \& FM JAKSIC (2008a) How general are global trends in biotic homogenization? Floristic tracking in Chile, South America. Global Ecology and Biogeography 17: 524-531.

CASTRO SA \& FM JAKSIC (2008b) Patterns of turnover and floristic similarity show a non-random distribution of naturalized flora in Chile, South America. Revista Chilena de Historia Natural 81: 111-121.

CAVENDER-BARES J, K KOZAK, P FINE \& S KEMBEL (2009) The merging of community ecology and phylogenetic biology. Ecology Letters 12: 693-715

CHESSON P (2000) Mechanisms of maintenance of species diversity. Annual Review of Ecology and Systematics 31: 343-366.

CONAMA (2003) Estrategia nacional de biodiversidad. Comisión Nacional del Medio Ambiente, Santiago, Chile.

D’ANTONIO CM \& PM VITOUSEK (1992) Biological invasions by exotic grasses, the grasses, the grass/fire cycle and global change. Annual Review of Ecology and Systematics 23: 63-87.

DAEHLER CC (1988) The taxonomic distribution of invasive angiosperm plants: Ecological insights and comparison to agricultural weeds. Biological Conservation 84: 167-180.

DAEHLER CC (2001) Darwin's naturalization hypothesis revisited. American Naturalist 158: 324-330.

DARWIN C (1859) On the origin of species. J. Murray, London.

DAVIS MA (2009) Invasion biology. Oxford University Press, New York.

DI CASTRI F (1989) History of biological invasions with special emphasis on the Old World. En: Drake JR, HA Mooney, F Di Castri, RH Groves, FJ Kruger, M Rejmánek \& M Williamson (eds) Biological invasions: A global perspective: 1-30. Scientific committee on problems of the environment (SCOPE). John Wiley \& Son, New York.

DIEZ JM, JJ SULLIVAN, PE HULME, G EDWARDS \& RP DUNCAN (2008) Darwin's naturalization conundrum: Dissecting taxonomic patterns of species invasions. Ecology Letters 11: 674-681.

DIEZ JM, PA WILLIAMS, RP RANDALL, JJ SULLIVAN, PE HULME \& RP DUNCAN (2009) Learning from failures: Testing broad taxonomic hypotheses about plant naturalization. Ecology Letters 12: 1174-1183.

DUNCAN RP \& PA WILLIAMS (2002) Darwin's 
naturalization hypothesis challenged. Nature 417: 608-609.

FIGUEROA JA, SA CASTRO, PA MARQUET \& FM JAKSIC (2004) Exotic plant invasions to the Mediterranean region of Chile: Causes, history and impacts. Revista Chilena de Historia Natural 77: 465-483.

GROVES RH (1991) Biogeography of Mediterranean plant invasions. En: Groves RH \& F Di Castri (eds) Biogeography of Mediterranean invasions: 427-438. Cambridge University Press, Cambridge, UK.

HUBBELL SP (2001) A unified neutral theory of biodiversity and biogeography. Princeton University Press, Princeton, New York.

HULME PE (2009) Relative roles of life-form, land use and climate in recent dynamics of alien plant distributions. Weed Research 49: 19-28.

INSTITUTO GEOGRÁFICO MILITAR (2010) Atlas geográfico para la educación. I.G.M. de Chile. Santiago, Chile.

JAKSIC FM (1998) Vertebrate invaders and their ecological impacts in Chile. Biodiversity and Conservation 7: 1427-1445.

JIANG L, J TAN \& Z PU (2010) An experimental test of Darwin's naturalization hypothesis. The American Naturalist 175: 415-423.

KOLAR CS \& DM LODGE (2001) Progress in invasions biology: Predicting invaders. Trends in Ecology and Evolution 16: 199-204.

LAMBDON PW \& PE HULME (2006) How strongly do interactions with closely-related native species influence plant invasions? Darwin's naturalization hypothesis assessed on Mediterranean islands. Journal of Biogeography 33: 1116-1125.

LOCKWOOD JL, MF HOOPES \& MP MARCHETTI (2007) Invasion ecology. Blackwell Publishing, Oxford, UK.

MACDOUGALL AS, B GILBERT \& JM LEVINE (2009) Plant invasions and the niche. Journal of Ecology 97: 609-615.

MACK RN (1996) Predicting the identity and fate of plant invaders: Emergent and emerging approaches. Biological Conservation 78: 107-121.

MACK RN, D SIMBERLOFF, WM LONSDALE, H EVANS, M CLOUT \& FA BAZZAZ (2000) Biotic invasions, causes, epidemiology, global consequences, and control. Ecological Applications 10: 689-710.

MARTICORENA C \& M QUEZADA (1985) Catálogo de la flora vascular de Chile. Gayana Botánica 42: 5-157.

MATTHEI O (1995) Manual de las malezas que crecen en Chile. Alfabeta Impresores, Santiago, Chile.

MAYFIELD M \& J LEVINE (2010) Opposing effects of competitive exclusion on the phylogenetic structure of communities. Ecology Letters 13: 1085-1093.

MCKINNEY ML (2006) Correlated non-native species richness of birds, mammals, herptiles and plants: Scale effects of area, human population and native plants. Biological Invasions 8: 415-425.

MITCHELL CE, AA AGRAWAL, JD BEVER, GS GILBERT, RA HUFBAUER et al. (2006) Biotic interactions and plant invasions. Ecology Letters 9: 726-740.

MONTENEGRO G, S TEILLIER, P ARCE \& V POBLETE (1991) Introductions of plants and animals into the Mediterranean-type climate area of Chile. En: Groves RH \& F Di Castri (eds) Biogeography of Mediterranean invasions: 103-114. Cambridge
University Press, Cambridge, UK.

PROCHEŞ S, JRU WILSON, DM RICHARDSON \& M REJMANEK (2008) Searching for phylogenetic pattern in biological invasions. Global Ecology and Biogeography 17: 5-10.

PYŠEK P, J SADLO, B MANDÁK \& V JAROSIK (2003) Czech alien flora and the historical pattern of its formation: What came first to central Europe? Oecologia 135: 122-130.

PYŠEK P \& DM RICHARDSON (2006) The biogeography of naturalization in alien plants. Journal of Biogeography 33: 2040-2050.

R DEVELOPMENT CORE TEAM (2010) R: A language and environment for statistical computing. $\mathrm{R}$ Foundation for Statistical Computing, Vienna, Austria. ISBN 3-900051-07-0. URL: http://www.rproject.org (acceso Diciembre 11, 2010).

REJMÁNEK M \& DM RICHARDSON (1996) What attributes make some plant species more invasive? Ecology 77: 1655-1660.

REJMÁNEK M (2000) Invasive plants: Approaches and predictions. Austral Ecology 25: 497-506.

RICCIARDI A \& SK ATKINSON (2004) Distinctiveness magnifies the impact of biological invaders in aquatic ecosystems. Ecology Letters 7:781-784.

RICCIARDI A \& M MOTTIAR (2006) Does Darwin's naturalization hypothesis explain fish invasions? Biological Invasions 8: 1403-1407.

RICHARDSON D, P PYŠEK, M REJMANEK, M BARBOUR, F PANETTA \& C WEST (2000) Naturalization and invasion of alien plants: Concepts and definitions. Diversity and Distributions 6: 93-107.

ROZENFIELDS ACF \& R MACKENZIE (1999) The weed invasion in Tasmania in the 1870s: Knowing the past to predict the future. En: Bishop AC, M Boersma \& C Barnes (eds) $12^{\text {th }}$ Australian weed conference papers and proceedings: 581-583. CSIRO Publications, Hobart, Tasmania.

SAKAI AK, FW ALLENDORF, JS HOLT, DM LODGE, J MOLOFSKY et al. (2001) The population biology of invasive species. Annual Review of Ecology and Systematics 32: 305-332.

SAX DF (2001) Latitudinal gradients and geographic ranges of exotic species: Implications for biogeography. Journal of Biogeography 28: $139-150$

SAX DF, JJ STACHOWICZ, JH BROWN, JF BRUNO, MN DAWSON et al. (2007) Ecological and evolutionary insights from species invasions. Trends in Ecology \& Evolution 22: 465-471.

SCHAEFER H, O HARDY, L SILVA, T BARRACLOUGH \& V SAVOLAINEN (2011) Testing Darwin's naturalization hypothesis in the Azores. Ecology Letters 14: 389-396

SHIGESADA N \& K KAWASAKI (2001) Biological invasions: Theory and practice. Oxford University Press, Oxford, UK.

STRAUSS SY, CO WEBB \& N SALAMIN (2006) Exotic taxa less related to native species are more invasive. Proceedings of the National Academy of Sciences USA 103: 5841-5845.

THEBAUD C \& D SIMBERLOFF (2001) Are plants really larger in their introduced ranges? American Naturalist 157: 231-236.

THEOHARIDES KA \& JS DUKES (2007) Plant invasion across space and time: Factors affecting nonindigenous species success during four stages of invasion. New Phytologist 176: 256-273 
THUILLER W, L GALLIEN, I BOULANGEAT, F DE BELLO, T MÜNKEMÜLLER, C ROQUET \& S LAVERGNE (2010) Resolving Darwin's naturalization conundrum: A quest for evidence. Diversity and Distributions 16: 1-15.

VILÁ M, I BARTOMEUS, AC DIETZSCH, T PETANIDOU, I STEFFAN-DEWENTER, JC STOUT \& T TSCHEULIN (2009) Invasive plant integration into native plant-pollinator networks across Europe. Proceedings of the Royal Society of London B 276: 3887-3893.

VIRGINIE M \& A BÉCHET (2010) From adaptive management to adjustive management: A pragmatic account of biodiversity values. Conservation Biology 24: 966-973

VITOUSEK PM (1994) Beyond global warming: Ecology and global change. Ecology 75: 1861-1876.
VITOUSEK PM, CM D'ANTONIO, LL LOOPE, M REJMÁNEK \& R WESTBROOKS (1997) Introduced species: A significant component of human-caused global change. New Zealand Journal of Ecology 21: 1-16.

WIENS JJ \& CH GRAHAM (2005) Niche conservatism: Integrating evolution, ecology, and conservation biology. Annual Review of Ecology, Evolution and Systematics 36: 519-539.

WILLIAMSON M (1996) Biological invasions. Chapman \& Hall, London, UK.

WU SH, SM CHAW \& M REJMÁNEK (2003) Naturalized Fabaceae (Leguminosae) species in Taiwan: The first approximation. Botanical Bulletin of Academia Sinica 44: 59-66.

Editor Asociado: Ernesto Gianoli

Recibido el 21 de septiembre de 2011; aceptado el 18 de noviembre de 2011 